PROCEEDINGS OF THE

AMERICAN MATHEMATICAL SOCIETY

Volume 137, Number 6, June 2009, Pages 2157-2167

S 0002-9939(09)09729-9

Article electronically published on February 4, 2009

\title{
SPANNING TREES AND KHOVANOV HOMOLOGY
}

\author{
ABHIJIT CHAMPANERKAR AND ILYA KOFMAN \\ (Communicated by Alexander N. Dranishnikov)
}

\begin{abstract}
The Jones polynomial can be expressed in terms of spanning trees of the graph obtained by checkerboard coloring a knot diagram. We show that there exists a complex generated by these spanning trees whose homology is the reduced Khovanov homology. The spanning trees provide a filtration on the reduced Khovanov complex and a spectral sequence that converges to its homology. For alternating links, all differentials on the spanning tree complex are zero and the reduced Khovanov homology is determined by the Jones polynomial and signature. We prove some analogous theorems for (unreduced) Khovanov homology.
\end{abstract}

\section{INTRODUCTION}

For any diagram of an oriented link $L$, Khovanov 3 constructed bigraded abelian groups $H^{i, j}(L)$ whose bigraded Euler characteristic gives the Jones polynomial $V_{L}(t)$ :

$$
\chi\left(H^{*, *}\right)=\sum_{i, j}(-1)^{i} q^{j} \operatorname{rank}\left(H^{i, j}\right)=\left(q+q^{-1}\right) V_{L}\left(q^{2}\right) .
$$

Khovanov's homology groups turn out to be stronger invariants than the Jones polynomial. For knots, Khovanov also defined a reduced homology $\widetilde{H}^{i, j}(L)$ whose bigraded Euler characteristic is $q^{-1} V_{L}\left(q^{2}\right)$ [4].

The Jones polynomial has an expansion in terms of spanning trees of the Tait graph, obtained by checkerboard coloring a given link diagram [9]. Every spanning tree contributes a monomial to the Jones polynomial, and for alternating knots, the number of spanning trees is exactly the $L^{1}$-norm of the coefficients of the Jones polynomial. These monomials are Kauffman brackets of certain twisted unknots (Theorem 2).

We show that the reduced Khovanov complex $\widetilde{C}(D)$ retracts to a spanning tree complex, whose generators correspond to spanning trees of the Tait graph (Theorem 3). The main idea is that a spanning tree corresponds to a twisted unknot $U$, and $\widetilde{C}(U)$ is contractible, providing a deformation retract of $\widetilde{C}(D)$. This extends to (unreduced) Khovanov homology (Theorem 4). The proof does not provide an intrinsic description of the differential on spanning trees. From a partial order on

Received by the editors May 24, 2007, and, in revised form, August 12, 2008.

2000 Mathematics Subject Classification. Primary 57M25.

The first author was supported by NSF grant DMS-0455978.

The second author was supported by grants NSF DMS-0456227 and PSC-CUNY 60046-3637. 
spanning trees, we get a filtration of $\widetilde{C}(D)$ and a spectral sequence that converges to $\widetilde{H}(D)$ (Theorem 5 ).

A knot $K$ is alternating if and only if all the spanning trees are in one row of the spanning tree complex and, hence, all differentials on the spanning tree complex are zero. We give a simple new proof of the fact that for alternating links $\widetilde{H}(K)$ is determined by its Jones polynomial and signature (Theorem 7 ). We also give simple new proofs for theorems in [5, 1, 6] on the support of Khovanov homology of alternating and $k$-almost alternating knots (Theorem 8).

Wehrli independently gave a spanning tree model for Khovanov homology in [13].

\section{Spanning trees And TWISted UnKNots}

There is a 1-1 correspondence between connected link diagrams $D$ and connected planar graphs $G$ with signed edges. $G$ is obtained by checkerboard coloring complementary regions of $D$, assigning a vertex to every shaded region, an edge to every crossing, and a + or - sign to every edge such that for a positive edge, the $A$-smoothing joins the shaded regions. The signs are all equal if and only if $D$ is alternating. $G$ is called the Tait graph of $D$. Thistlethwaite 9 described the following expansion of the Jones polynomial in terms of spanning trees of the Tait graph.

Fix an order on the edges of $G$. For every spanning tree $T$ of $G$, each edge $e$ of $G$ has an activity with respect to $T$, as originally defined by Tutte. If $e \in T$, $\operatorname{cut}(T, e)$ is the set of edges that connect $T \backslash e$. If $f \notin T, \operatorname{cyc}(T, f)$ is the set of edges in the unique cycle of $T \cup f$. Note that $f \in \operatorname{cut}(T, e)$ if and only if $e \in \operatorname{cyc}(T, f)$. An edge $e \in T$ is called internally active with respect to $T$ if it is the lowest edge in its cut; otherwise it is called internally inactive. An edge $e \notin T$ is externally active with respect to $T$ if it is the lowest edge in its cycle; otherwise it is externally inactive. Each edge has one of eight possible activities, depending on whether (i) $e \in T$ or $e \notin T$, (ii) $e$ is active (live) or inactive (dead), (iii) $e$ has $\mathrm{a}+$ or $-\operatorname{sign}$. Let $L, D, \ell$, and $d$ denote a positive edge that is internally active and internally inactive, externally active and externally inactive, respectively. Let $\bar{L}, \bar{D}, \bar{\ell}, \bar{d}$ denote activities for a negative edge. Each edge $e$ of $G$ is assigned a monomial $\mu_{e} \in \mathbb{Z}\left[A^{ \pm 1}\right]$, as in Table 1 (last row). Let $\mu(T)=\prod_{e \in G} \mu_{e}$.

Theorem 1 (9]). Let $D$ be any connected link diagram, and let $G$ be its Tait graph with any order on its edges. Then the Kauffman bracket satisfies $\langle D\rangle=$ $\sum_{T \subset G} \mu(T)$.

TABLE 1. Activity word for a spanning tree determines a twisted unknot

\begin{tabular}{cc|cc|cc|cc}
$L$ & $D$ & $\ell$ & $d$ & $\bar{L}$ & $\bar{D}$ & $\bar{\ell}$ & $\bar{d}$ \\
\hline- & $A$ & + & $B$ & + & $B$ & - & $A$ \\
$\searrow$ & $\nearrow$ & $\searrow$ & )( & $\searrow$ & $\nearrow$ & $\nearrow$ & )( \\
\hline$-A^{-3}$ & $A$ & $-A^{3}$ & $A^{-1}$ & $-A^{3}$ & $A^{-1}$ & $-A^{-3}$ & $A$
\end{tabular}

Using the edge order, we write an activity word for each spanning tree $T$ using the eight letters for its edge activities. $T$ is given by the capital letters of the word. A twisted unknot $U$ is obtained from the round unknot by using only Reidemeister I moves. 
Lemma 1. Given an activity word for a spanning tree $T$, changing the crossings of $D$ according to Table 1 for dead edges while leaving the crossings unchanged for live edges gives a twisted unknot $U(T)$.

Proof. We need to show that every crossing of $U(T)$ can be undone by a Reidemeister I move. Given $T$, we can obtain $U(T)$ as follows: first draw $U$ as if all edges in $G$ are dead, i.e. as a regular neighborhood of $T$ which is a round unknot up to planar isotopy. Now for each $e$ not in $T$, we put a crossing there only if $e$ is live, so that this will be the only crossing in $\operatorname{cyc}(T, e)$, which is a cycle in a planar graph. Hence $U$ remains a round unknot after a Reidemeister I move; similarly for all live edges $e$ not in $T$. For all live edges $f$ in $T$, redo this argument for the dual tree $T^{*}$ using $\operatorname{cut}(T, f)=\operatorname{cyc}\left(T^{*}, f^{*}\right)$. Therefore, $U(T)$ is isotopic in the plane to the round unknot after a sequence of Reidemeister I moves.

If $U$ is a partial smoothing of $D$, let $\sigma(U)=\# A$-smoothings - \#B-smoothings.

Theorem 2. Let $D$ be any connected link diagram, and let $G$ be its Tait graph with any order on its edges. There exists a partial skein resolution tree $\mathcal{T}$, whose leaves are twisted unknots that correspond to spanning trees of $G$. If $U$ corresponds to $T$, then $\mu(T)=A^{\sigma(U)}(-A)^{3 w(U)}$.

Proof. To construct $\mathcal{T}$, we order the crossings of $D$ in reverse order to the edges of $G$. Let the root of $\mathcal{T}$ be $D$. A crossing is called nugatory if either its $A$ or its $B$ smoothing disconnects the diagram. We smooth the crossings of $D$ in order, such that at every branch we leave nugatory crossings unsmoothed and go to the next crossing. Stop when all subsequent crossings are nugatory. Since a diagram is a twisted unknot if and only if all crossings are nugatory, the leaves of $\mathcal{T}$ are twisted unknots.

From any twisted unknot $U$ in $\mathcal{T}$, we can obtain a spanning tree $T(U)$ of $G$ by using Table 1, where the signs below the live edges indicate the writhe of the crossing. By Lemma 1, $U=U(T(U))$. Each live edge determines the writhe of its crossing in $U$, hence $\mu(T)=A^{\sigma(U)}(-A)^{3 w(U)}$.

The activity word for $T$ determines a partial smoothing $U(T)$. Live edges are not smoothed, denoted below by $*$.

Definition 1. Let $D$ be any connected link diagram with $n$ ordered crossings. For any spanning trees $T, T^{\prime}$ of $G$, let $\left(x_{1}, \ldots, x_{n}\right)$ and $\left(y_{1}, \ldots, y_{n}\right)$ be the corresponding partial smoothings of $D$. We define a relation $T>T^{\prime}$ or, equivalently, $\left(x_{1}, \ldots, x_{n}\right)>\left(y_{1}, \ldots, y_{n}\right)$ if, for each $i, y_{i}=A$ implies $x_{i}=A$ or $*$ and there exists an $i$ such that $x_{i}=A$ and $y_{i}=B$. By Proposition 1, the transitive closure of this relation gives a partial order, also denoted by $>$. We define $P(D)$ to be the poset of spanning trees of $G$ with this partial order.

Proposition 1. If $T>\cdots>T^{\prime}$, then $T \neq T^{\prime}$.

Proof. We can draw $\mathcal{T}$ in such a way that the $A$-smoothing of $c_{i}$ is $2^{-i}$ units to the left of its parent node and the $B$-smoothing of $c_{i}$ is $2^{-i}$ units to the right. For any $T>\cdots>T^{\prime}, T$ is to the left of $T^{\prime}$.

Note that $P(D)$ always has a unique maximal tree and a unique minimal tree whose partial smoothings contain the all- $A$ and all- $B$ Kauffman states, respectively. 


\section{SPANNING TREE COMPLEX}

Every spanning tree $T$ of a Tait graph $G$ with ordered edges corresponds to an activity word, which in turn corresponds to a twisted unknot $U$. Let $w(U)$ denote the writhe of $U$, let $V(G)$ denote the number of vertices of $G$ and let $E_{ \pm}(G)$ denote the number of positive or negative edges of $G$. Given $D$, we require that the checkerboard coloring be chosen such that $E_{+}(G) \geq E_{-}(G)$.

Definition 2. Let $D$ be a connected knot diagram with ordered crossings, and let $G$ be its ordered Tait graph. For any spanning tree $T$ of $G$, we define bigradings

$$
u(T)=-w(U)=\# L-\# \ell-\# \bar{L}+\# \bar{\ell} \text { and } v(T)=E_{+}(T)=\# L+\# D .
$$

Define $\mathcal{C}(D)=\bigoplus_{u, v} \mathcal{C}_{v}^{u}(D)$, where $\mathcal{C}_{v}^{u}(D)=\mathbb{Z}\langle T \subset G \mid u(T)=u, v(T)=v\rangle$.

Define $\mathcal{U C}(D)=\bigoplus_{u, v}\left(\mathcal{C}_{v}^{u}(D)+\overline{\mathcal{C}}_{v+1}^{u+2}(D)\right)$, where $\overline{\mathcal{C}}_{v+1}^{u+2}(D) \cong \mathcal{C}_{v}^{u}(D)$.

Proposition 2. For any differential $\partial: \mathcal{C}_{v}^{u} \rightarrow \mathcal{C}_{v-1}^{u-1}$, the Jones polynomial can be expressed as the graded Euler characteristic of $\{\mathcal{C}(D), \partial\}$ and of $\{\mathcal{U C}(D), \partial\}$ :

$$
\begin{aligned}
V_{D}(t) & =(-1)^{w(D)} t^{\frac{3 w(D)+k}{4}} \chi(\mathcal{C}(D)), \\
\left(t^{1 / 2}+t^{-1 / 2}\right) V_{D}(t) & =(-1)^{w(D)} t^{\frac{3 w(D)+k+2}{4}} \chi(\mathcal{U C}(D)),
\end{aligned}
$$

where $w(D)$ is the writhe of $D$ and $k=E_{+}(G)-E_{-}(G)+2(V(G)-1)$.

Proof. Let $G$ be the Tait graph of $D$, and let $T$ be any spanning tree of $G$. By Table 1, the weight of $T$ is given as follows:

$$
L^{p} D^{q} \ell^{r} d^{s} \bar{L}^{x} \bar{D}^{y} \bar{\ell}^{z} \bar{d}^{w} \quad \Rightarrow \quad \mu(T)=(-1)^{p+r+x+z} A^{-3 p+q+3 r-s+3 x-y-3 z+w} .
$$

Since $T$ is a tree, we have $p+q+x+y=V(G)-1$ and $r+s+z+w=E(G)-V(G)+1$. Also $p+q+r+s=E_{+}(G)$ and $x+y+z+w=E_{-}(G)$. Let $k=E_{+}(G)-E_{-}(G)+$ $2(V(G)-1)$. Since $u=p-r-x+z$ and $v=p+q, \mu(T)=(-1)^{u} A^{-4(u-v)-k}$. Hence

$$
\langle D\rangle=\sum_{T \subset G} \mu(T)=A^{-k} \sum_{u}(-1)^{u} \sum_{v} A^{-4(u-v)}\left|\mathcal{C}_{v}^{u}\right| .
$$

For $t=A^{-4}, V_{D}(t)=(-A)^{-3 w(D)}\langle D\rangle$, so the first result follows. We also have

$$
\begin{aligned}
\chi(\mathcal{U C}(D)) & =\sum_{u}(-1)^{u} \sum_{v} t^{(u-v)}\left(\left|\mathcal{C}_{v}^{u}\right|+\left|\bar{C}_{v+1}^{u+2}\right|\right) \\
& =\sum_{u, v}(-1)^{u} t^{(u-v)}\left|\mathcal{C}_{v}^{u}\right|+t^{-1} \sum_{u, v}(-1)^{u+2} t^{((u+2)-(v+1))}\left|\bar{C}_{v+1}^{u+2}\right| \\
& =\left(1+t^{-1}\right) \sum_{u, v}(-1)^{u} t^{(u-v)}\left|\mathcal{C}_{v}^{u}\right| \doteq\left(t^{1 / 2}+t^{-1 / 2}\right) V_{D}(t) .
\end{aligned}
$$

The final equality is the second result up to multiplication by $(-1)^{w(D)} t^{\frac{3 w(D)+k+2}{4}}$.

Let $\widetilde{C}(D)=\left\{\widetilde{C}^{i, j}(D), \partial\right\}$ denote the reduced Khovanov complex as in 12, 11, with $\widetilde{H}^{i, j}(\bigcirc)=\mathbb{Z}^{(0,-1)}$ where $\bigcirc$ denotes the round unknot. For chain complexes $X$ and $Y, X$ is a deformation retract of $Y$ if there exist chain maps $r: Y \rightarrow X$ and $f: X \rightarrow Y$ such that $r \circ f=i d_{X}$, and a chain homotopy $F: Y \rightarrow Y$ such that $\partial_{Y} F+F \partial_{Y}=i d_{Y}-f \circ r$. The map $r$ is called a retraction. 

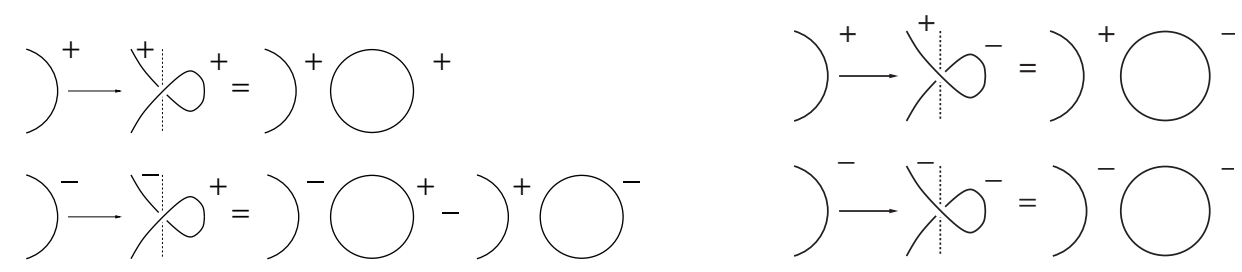

FIgURE 1. Jacobsson rules for a positive and negative twist

Theorem 3. For a knot diagram $D$, there exists a spanning tree complex $\mathcal{C}(D)=$ $\left\{\mathcal{C}_{v}^{u}(D), \partial\right\}$ with $\partial$ of bi-degree $(-1,-1)$ that is a deformation retract of $\widetilde{C}(D)$. In particular, if $w$ is the writhe of $D$ and $k=E_{+}(G)-E_{-}(G)+2(V(G)-1)$, then

(1) $\widetilde{H}^{i, j}(D ; \mathbb{Z}) \cong H_{v}^{u}(\mathcal{C}(D) ; \mathbb{Z}), \quad u=j-i-w+1, v=j / 2-i-(w-k-2) / 4$.

Theorem 4. There exists an unreduced spanning tree complex $\mathcal{U C}(D)=$ $\left(\mathcal{U C}_{v}^{u}(D), \partial\right)$ with $\partial$ of bi-degree $(-1,-1)$ that is a deformation retract of the (unreduced) Khovanov complex. In particular, with indices related as in $(\mathbb{1}), H^{i, j}(D ; \mathbb{Z}) \cong$ $H_{v}^{u}(\mathcal{U C}(D) ; \mathbb{Z})$.

For a twisted unknot $U, \widetilde{C}(U)$ is contractible. Its homology is generated by a single generator in degree $(i, j)=(0,-1)$, which is given by iterating the four Jacobsson rules: Starting from $\bigcirc$, by a sequence of positive and negative twists, we obtain $U$. Figure 1 indicates how to change the enhanced state for each twist, starting with the round unknot enhanced by a + sign, $\bigcirc^{+}$, which generates $\widetilde{C}(\bigcirc) \cong$ $\mathbb{Z}^{(0,-1)}$.

Definition 3. For any twisted unknot $U$, we define its fundamental cycle $Z_{U} \in$ $\widetilde{C}(U)$ to be the linear combination of maximally disconnected enhanced states of $U$ given by the Jacobsson rules. Let $f_{U}: \widetilde{C}(\bigcirc) \rightarrow \widetilde{C}(U)$ be defined by $f_{U}\left(\bigcirc^{+}\right)=Z_{U}$.

Let $w(U)$ be the writhe of $U$, let $\sigma$ be the difference of $A$-smoothings and $B$ smoothings, and let $\tau$ be the difference of positive and negative enhancements, as in 12. By Figure 1, the Jacobsson rules have the following effect for each added twist:

$$
\begin{array}{lll}
\text { Positive twist: } & w \rightarrow w+1 \quad \sigma \rightarrow \sigma+1 \quad \tau \rightarrow \tau+1 \\
\text { Negative twist: } & w \rightarrow w-1 \quad \sigma \rightarrow \sigma-1 & \tau \rightarrow \tau-1
\end{array}
$$

The grading for any enhanced state in $\widetilde{C}^{i, j}(U)$ is given by $i=(w-\sigma) / 2$ and $j=i+w-\tau$, which are preserved under the Jacobsson rules. By Lemma 4 below, $f_{U}$ is a grading-preserving chain homotopy. Let $\iota: \widetilde{C}(U) \rightarrow \widetilde{C}(D)$ be the inclusion of enhanced states of $U$ into enhanced states of $D$ given by the grading shifts $\iota\left(s^{i, j}\right)=s^{i^{\prime}, j^{\prime}}$, where $i^{\prime}=i+\frac{w(D)-w(U)-\sigma(U)}{2}$ and $j^{\prime}=j+\frac{3(w(D)-w(U))-\sigma(U)}{2}$. For any spanning tree $T_{k}$, we define $\widetilde{U}_{k}=\iota\left(\widetilde{C}\left(U\left(T_{k}\right)\right)\right) \subset \widetilde{C}(D)$.

Proof of Theorem 3. Fix any order on the crossings of $D$ and a basepoint on $D$ away from the crossings. We embed $\mathcal{C}(D)$ into $\widetilde{C}(D)$ as bigraded groups. For each generator $T \in \mathcal{C}_{v}^{u}(D)$, let $U=U(T)$. Let $\phi: \mathcal{C}(D) \rightarrow \widetilde{C}(D)$ be defined by $\phi(T)=\iota\left(Z_{U(T)}\right)$.

For given $(u(T), v(T))$, we compute the $(i, j)$ degree of $\phi(T)$ in $\widetilde{C}(D)$. Let $\sigma$ and $\sigma_{U}$ denote the signatures $(\# A-\# B)$ for enhanced states in $\widetilde{C}(D)$ and $\widetilde{C}(U)$, 
respectively. Since $Z_{U(T)} \in \widetilde{C}^{0,-1}(U)$, we have $w(U)-\sigma_{U}=0$ and $w(U)-\tau=$ -1 . Since $u(T)=-w(U)$, we have $\sigma_{U}=-u(T)$ and $\tau=1-u(T)$. Since $U$ is obtained from $D$ by smoothing all crossings on dead edges, using the notation of Proposition 2, $\sigma=\sigma_{U}+(q-s-y+w)$. Since $q-s-y+w=-u+4 v-k(D), \tau$ and $\sigma$ of $Z_{U(T)}$ are $\tau=1-u(T)$ and $\sigma=-2 u(T)+4 v(T)-k(D)$. Therefore, $\phi(T)$ has the following $(i, j)$ degree in $\widetilde{C}(D)$ : If $w=w(D)$ and $k=k(D)$, then

(2) $i=\frac{w-\sigma}{2}=u-2 v+\frac{w+k}{2}$ and $j=i+w-\tau=2 u-2 v+\frac{3 w+k-2}{2}$.

Solving for $u$ and $v$, we obtain (11).

We now order the spanning trees of $G$ as $T_{k}, 1 \leq k \leq s$, such that if $T_{k_{1}}>T_{k_{2}}$, then $k_{1}>k_{2}$. We proceed by a sequence of elementary collapses of each unknot's complex to its fundamental cycle, starting from the minimal tree. Lemma 5 provides the crucial fact that any elementary collapse in $\widetilde{U}_{k}$ does not change incidence numbers in $\widetilde{U}_{c}$ for any $c \neq k$. This fact permits us to repeatedly apply Lemma 4 . Starting with $C_{0}=\widetilde{C}(D)$, we get a sequence of complexes $C_{k}, 0 \leq k \leq s$, and retractions $r_{k}: C_{0} \rightarrow C_{k}$. Each $C_{k+1}$ is obtained from $C_{k}$ via a sequence of elementary collapses by deleting all generators in $r_{k}\left(\widetilde{U}_{k}\right)$ except the fundamental cycle. Finally, $C_{s}$ is a complex whose generators are in one-to-one correspondence with the spanning trees of $G$ and such that $\widetilde{H}^{i, j}\left(C_{s}\right) \cong \widetilde{H}^{i, j}(D)$.

The map $r_{s} \circ \phi: \mathcal{C}_{v}^{u}(D) \rightarrow C_{s}$ is a graded isomorphism of groups, with the indices related by (1). The induced differential on the spanning tree complex $\mathcal{C}_{v}^{u}(D)$ now gives $H_{v}^{u}(\mathcal{C}(D)) \cong \widetilde{H}^{i, j}(D)$ with the indices related by (1). In the version of Khovanov homology in [12, the differential on $\widetilde{C}(D)$ has bi-degree $(1,0)$, so by (1) the differential on $\mathcal{C}_{v}^{u}(D)$ has bi-degree $(-1,-1)$. The retraction from the reduced Khovanov complex to the spanning tree complex is given by

$$
r=\left(r_{s} \circ \phi\right)^{-1} \circ r_{s}: \widetilde{C}^{i, j}(D) \rightarrow \mathcal{C}_{v}^{u}(D)
$$

where $r\left(\widetilde{U}_{k}\right)=T_{k}, r \circ \phi=i d$, and the indices are related by (1).

For a complex $(C, \partial)$ over $\mathbb{Z}$, we say $x$ is incident to $y$ in $(C, \partial)$ if $\langle\partial x, y\rangle \neq 0$; their incidence number is $\langle\partial x, y\rangle$.

Lemma 2. The differential $\partial$ on $\widetilde{C}(D)$ respects the partial order in Definition 1 ; (i) If $\langle\partial x, y\rangle \neq 0$ for any $x \in \widetilde{U}_{1}$ and $y \in \widetilde{U}_{2}$, then $T_{1}>T_{2}$. (ii) If $T_{1}$ and $T_{2}$ are not comparable or $T_{2}>T_{1}$, then $\langle\partial x, y\rangle=0$ for all $x \in \widetilde{U}_{1}$ and $y \in \widetilde{U}_{2}$.

Proof. If $\langle\partial x, y\rangle \neq 0$, then any partial smoothing that contains these states satisfies $\left(x_{1}, \ldots, x_{n}\right)>\left(y_{1}, \ldots, y_{n}\right)$ as in Definition 1

Lemma 3 (Elementary collapse). Let $(C, \partial)$ be a finitely generated chain complex over $\mathbb{Z}$. Let $x, y$ be generators such that $x \in C_{k}$ and $y \in C_{k-1}$ with $\langle\partial x, y\rangle= \pm 1$. Then there exists a complex $\left(C^{\prime}, \partial\right)$ such that $C^{\prime}$ is generated by the same generators as $C$ except for $x$ and $y$, and there is a retraction $r: C \rightarrow C^{\prime}$.

Proof. Fix bases $E_{n}$ of $C_{n}$ with $E_{k-1}=\left\{y, y_{1}, \ldots, y_{d_{k-1}}\right\}, E_{k}=\left\{x, x_{1}, \ldots, x_{d_{k}}\right\}$. For $n \geq 0$, define $r_{n}: C_{n} \rightarrow C_{n}$ as follows: For any $v \in C_{n}, r_{n}(v)=v$ if $n \neq k, k-1$,

$$
r_{n} v=v-\frac{\langle v, y\rangle}{\langle\partial x, y\rangle} \partial x \text { if } n=k-1, \quad \text { and } \quad r_{n} v=v-\frac{\langle\partial v, y\rangle}{\langle\partial x, y\rangle} x \text { if } n=k .
$$


Define $r: C \rightarrow C$ by $\left.r\right|_{C_{n}}=r_{n}$. Then $r$ is a chain map, and hence its image is a subcomplex. Let $\left(C^{\prime}, \partial\right)=(r(C), \partial)$. If $\partial x=\lambda y+Y$, with $\lambda= \pm 1$ and $\langle y, Y\rangle=0$, then for $i \geq 1, r_{k-1}\left(y_{i}\right)=y_{i}$ and $r_{k-1}(y)=-\lambda Y$. For $i \geq 1, r_{k}\left(x_{i}\right)=$ $x_{i}-\lambda\left\langle\partial x_{i}, y\right\rangle x$, and $r_{k}(x)=0$. It follows that $r: C \rightarrow C^{\prime}$ is a retraction.

Lemma 4. Let $U$ be a twisted unknot. There exists a sequence of elementary collapses, $r_{U}: \widetilde{C}(U) \rightarrow \widetilde{C}(\bigcirc)$, such that $r_{U} \circ f_{U}=i d$ and $f_{U} \circ r_{U} \simeq i d$.

Proof. In essence, this result follows from invariance of Khovanov homology under the first Reidemeister move 3 , but we explicitly provide the elementary collapses. The proof is by induction on the number of crossings of $U$. Suppose $U^{\prime}$ is obtained from $U$ by adding one kink and hence one crossing $c$. The markers below refer to $c$, and the signs to the enhancements near $c$ in the order that they appear in Figure1,

Positive twist. The $A$-smoothing of $U^{\prime}$ at $c$ results in a new loop; the $B$-smoothing does not. For every enhanced state $v^{+}$of $U$, collapse the pair $A^{+-} \rightarrow B^{+}$. For every enhanced state $v^{-}$of $U$, collapse the pair $A^{--} \rightarrow B^{-}$. By Lemma $3 r\left(A^{++}\right)=v^{+}$, and since $A^{-+} \rightarrow B^{+}$, we get $r\left(A^{-+}-A^{+-}\right)=v^{-}$.

Negative twist. The $B$-smoothing of $U^{\prime}$ at $c$ results in a new loop; the $A$ smoothing does not. For every enhanced state $v^{+}$of $U$, collapse the pair $A^{+} \rightarrow B^{++}$. For every enhanced state $v^{-}$of $U$, collapse the pair $A^{-} \rightarrow B^{-+}$. By Lemma 3 , $r\left(B^{+-}\right)=v^{+}$and $r\left(B^{--}\right)=v^{-}$.

Let $f: \widetilde{C}(U) \rightarrow \widetilde{C}\left(U^{\prime}\right)$ be the following map: For any $s \in \widetilde{C}(U)$, let $f(s)$ be the linear combination of states given by Figure 1. From the change in $w, \sigma$ and $\tau$, $f$ is grading-preserving. Moreover, $f$ is an iterated Jacobsson map: $f \circ f_{U}=f_{U^{\prime}}$. The elementary collapses above show that $\widetilde{C}(U) \stackrel{f}{\rightarrow} \widetilde{C}\left(U^{\prime}\right) \stackrel{r}{\rightarrow} \widetilde{C}(U)$ with $r \circ f=i d$ and $f \circ r \simeq i d$. Starting with $U=\bigcirc$, the result follows by induction.

Lemma 5. Let $D$ be a connected link diagram and let $G$ be its Tait graph. Let $T_{1}$ and $T_{2}$ be distinct spanning trees of $G$. Then in $\widetilde{C}(D)$, any elementary collapse as in Lemma 3 of $x_{1}, y_{1} \in \widetilde{U}_{1}$ will not change the incidence number between any $x_{2}, y_{2} \in \widetilde{U}_{2}$.

Proof. As in the proof of Lemma 3, $\left\langle\partial x_{1}, y_{1}\right\rangle=\lambda \in\{ \pm 1\}$. The image of $x_{2}$ after the elementary collapse of $x_{1}, y_{1}$ is $x_{2}^{\prime}=r\left(x_{2}\right)=x_{2}-\lambda\left\langle\partial x_{2}, y_{1}\right\rangle x_{1}$. Hence, $\left\langle\partial x_{2}^{\prime}, y_{2}\right\rangle=$ $\left\langle\partial x_{2}, y_{2}\right\rangle-\lambda\left\langle\partial x_{2}, y_{1}\right\rangle\left\langle\partial x_{1}, y_{2}\right\rangle$. By Lemma 2 if $T_{1}>T_{2}$, then $\left\langle\partial x_{2}, y_{1}\right\rangle=0$, and otherwise $\left\langle\partial x_{1}, y_{2}\right\rangle=0$. Thus, $\left\langle\partial x_{2}^{\prime}, y_{2}\right\rangle=\left\langle\partial x_{2}, y_{2}\right\rangle$.

Proof of Theorem 4. For (unreduced) Khovanov homology, $H^{i, j}(\bigcirc ; \mathbb{Z})=\mathbb{Z}^{0,1} \oplus$ $\mathbb{Z}^{0,-1}$. So the Khovanov complex for any twisted unknot $U$ is chain homotopic to the complex with two generators in degrees $(i, j)=(0, \pm 1)$. Hence, for every $T$, there are two fundamental cycles for $U(T)$ and two corresponding generators: $T_{+}$ in grading $(u(T), v(T))$ and $T_{-}$in grading $(u(T)+2, v(T)+1)$. Lemmas 2 , 4 and 5 now extend to the unreduced Khovanov complex, and the rest of the proof follows from that of Theorem 3 .

\section{Spanning tree filtration and spectral sequence}

The poset of spanning trees $P$ given in Definition 1, together with Proposition 1 and Lemma 2, provides a partially ordered filtration of $\widetilde{C}(D)$ indexed by $P$ : Let $\psi: P \rightarrow \widetilde{C}(D)$ be defined by $\psi(T)=+_{T \geq T_{i}} \widetilde{U}_{i}$. This defines a decreasing linearly 
ordered filtration on $\widetilde{C}(D)$ as follows. Let $\left\{S_{j}\right\}$ be the set of maximal descending ordered sequences of spanning trees in $P$, and let $T_{k}^{j}$ denote the $k$-th element of $S_{j}$, so that for all $j, T_{1}^{j}$ is the unique maximal tree in $P$. Define $F^{p} \widetilde{C}(D)=+_{j} \psi\left(T_{p}^{j}\right)$.

Theorem 5. For any knot diagram $D$, there is a spectral sequence $E_{r}^{*, *}$ that converges to the reduced Khovanov homology $\widetilde{H}^{*, *}(D ; \mathbb{Z})$ such that, as groups, $E_{1}^{*, *} \cong$ $\mathcal{C}_{*}^{*}(D)$, and the spectral sequence collapses for $r \leq c(D)$, where $c(D)$ is the number of crossings.

Proof. By Lemma 2, the differential on $\widetilde{C}(D)$ respects the filtration $\left\{F^{p} \widetilde{C}(D)\right\}$; i.e., $\partial F^{p} \subseteq F^{p}$. Hence this filtration determines a spectral sequence $\left\{E_{r}^{p, q}, d_{r}\right\}$ that converges to the reduced Khovanov homology. The associated graded module consists of submodules of $\widetilde{C}(D)$ in bijection with spanning trees:

$$
E_{0}^{p, *}=F^{p} \widetilde{C}(D) / F^{p+1} \widetilde{C}(D)=\bigoplus_{k} \widetilde{U}_{k}
$$

It follows from the filtration that for any $p$, if $\widetilde{U}_{1}, \widetilde{U}_{2} \subset E_{0}^{p, *}$, then $T_{1}$ and $T_{2}$ are not comparable in $P$. Hence, by Lemma $2(i i), d_{0}: E_{0}^{p, q} \rightarrow E_{0}^{p, q+1}$ satisfies $d_{0}\left(\widetilde{U}_{k}\right) \subset \widetilde{U}_{k}$ for every $k$. This implies that (4) is a direct sum of complexes $\widetilde{U}_{k}$. By Lemma 4 , each complex $\widetilde{U}_{k}$ has homology generated by $\phi\left(T_{k}\right)$. Therefore, $E_{1}$ is isomorphic as a group to the spanning tree complex:

$$
E_{1}^{*, *}=H^{*}\left(F^{p} / F^{p+1}, d_{0}\right)=\bigoplus_{k} H^{*}\left(\widetilde{U}_{k}\right) \cong \mathcal{C}_{*}^{*}(D)
$$

Since the length of any ordered sequence in $P$ is at most the number of crossings $c(D)$, it follows that the spectral sequence collapses for $r \leq c(D)$.

For field coefficients, Theorem 5 provides another proof of the fact that a differential exists on the spanning tree complex $\mathcal{C}(D)$ that makes it chain homotopic to $\widetilde{C}(D)$ :

Theorem 6. For coefficients in a field $\mathbb{F}$, there exists a differential on $\mathcal{C}(D)$ such that $\widetilde{H}^{*, *}(D ; \mathbb{F}) \cong H_{*}^{*}(\mathcal{C}(D) ; \mathbb{F})$.

Proof. By Lemma 4.5 in [7, there exists a unique filtered complex $C^{\prime}$ which is chain homotopic to $\widetilde{C}(D)$ and satisfies $C^{\prime} \cong H^{*}\left(F^{p} \widetilde{C}(D) / F^{p+1} \widetilde{C}(D)\right)$. Theorem 5 implies that $C^{\prime} \cong H^{*}\left(E_{0}^{*, *}\right) \cong E_{1}^{*, *} \cong \mathcal{C}(D)$.

\section{Alternating And almost alternating links}

We give simple new proofs of the theorems of Lee [5] and Shumakovitch $[8$ for the reduced Khovanov homology, using the spanning tree complex.

Theorem 7. The reduced Khovanov homology of an alternating knot is determined by its Jones polynomial and signature, and it has no torsion.

Proof. An alternating diagram $D$ can be checkerboard colored so that its Tait graph $G$ has all positive edges. For any spanning tree $T$ of $G, v(T)=E_{+}(T)=E(T)=$ $V(G)-1$. Since the $v$-grading is constant for all spanning trees, all the generators in the spanning tree complex $\mathcal{C}(D)$ are in one row. Since the differential on $\mathcal{C}(D)$ has degree $(-1,-1)$, it is trivial. Hence by Theorem 3 , $\widetilde{H}^{i, j}(D ; \mathbb{Z}) \cong H_{v}^{u}(\mathcal{C}(D) ; \mathbb{Z}) \cong$ $\mathcal{C}_{v}^{u}(D)$. Therefore, the homology has no torsion. The Betti numbers are determined 
by the Jones polynomial: If $c(D)$ is the number of crossings of $D$, then Proposition 2 implies that $\left|\mathcal{C}_{v}^{u}(D)\right|=a_{u-v+\frac{3 w(D)+c(D)+2 v}{4}}$, where $V_{D}(t)=\sum a_{n} t^{n}$ and we have used the relation $k(D)=E(G)+2\left(V^{4}(G)-1\right)=c(D)+2 v$. By [10], the signature of the knot is $\sigma(D)=\frac{c(D)-w(D)}{2}-\left|s_{B}\right|+1$, where $s_{B}(D)$ is the Kauffman state with all $B$ markers. Since $D$ is alternating, $\left|s_{B}(D)\right|=V(G)=v+1$. Therefore, $v=\frac{c(D)-w(D)}{2}-\sigma(D)$.

Remark 1. Using (2), the above proof implies that for non-split alternating links,

$$
j-2 i=2(V(G)-1)+\frac{w(D)-k(D)}{2}-1=v-\frac{c(D)-w(D)}{2}-1=-\sigma(D)-1
$$

A link is $k$-almost alternating if it has a non-nugatory diagram which is alternating after $k$ crossing changes but not after $k-1$ crossing changes. The bigraded homology of a link is $k$-thick if the nontrivial homology groups lie on $k$ adjacent lines. We give a simple new proof of theorems about the support of Khovanov homology for alternating and $k$-almost alternating links, which were obtained respectively by Lee [5] and Asaeda and Przytycki [1]. We proved a more general result in terms of ribbon graph genus in 2]. Another proof also appeared in Manturov 6].

Theorem 8. (i) The Khovanov homology of a non-split alternating link $L$ is at most 2-thick and lies on the lines $j-2 i=-\sigma(L) \pm 1$. Its torsion lies on the line $j-2 i=-\sigma(D)-1$. (ii) The Khovanov homology of a non-split $k$-almost alternating link $L$ is at most $(k+2)$-thick, and its reduced Khovanov homology is at most $(k+1)$-thick.

Proof. (i) For an alternating diagram $D, \mathcal{U C}(D)$ lies on two lines, $v=V(G)-1$ and $v=V(G)$. From Remark 1 the homology lies on the lines $j-2 i=-\sigma(D) \pm 1$. Moreover, since the differential on $\mathcal{U C}(D)$ has degree $(-1,-1)$, any torsion in the homology must lie on the line $j-2 i=-\sigma(D)-1$.

(ii) A $k$-almost alternating link or its mirror image has a Tait graph $G$ with exactly $k$ negative edges, so that $k \leq E(G) / 2$. For any spanning tree $T$ of $G$, $v(T)=E_{+}(T)$, so $v(T)$ takes at most $(k+1)$ values. Since $\mathcal{U C}(D)$ has at most $(k+2)$ rows, $H_{v}^{u}(\mathcal{U C}(D))$ has at most $(k+2)$ rows. The result now follows from Theorems 3 and 4 .

\section{EXAmple}

As an example we use a 4-crossing diagram of the trefoil. Here is the diagram $D$ and its Tait graph $G$. Below we show all the spanning trees of $G$ with their activity words, $(u, v)$-gradings and partial smoothings.
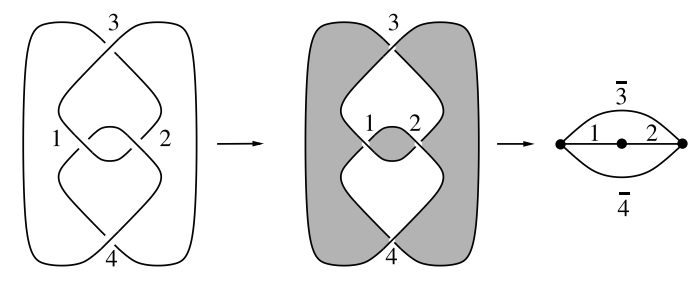


\begin{tabular}{|c|c|c|c|c|}
\hline & $\overline{3}$ & $\overline{3}$ & \\
\hline$T_{1}=\ell D \overline{D d}$ & $T_{2}=\ell D \overline{\ell D}$ & $T_{3}=L d \overline{D d}$ & $T_{4}=L d \overline{\ell D}$ & $T_{5}=L L \overline{d d}$ \\
\hline$(-1,1)$ & $(0,1)$ & $(1,1)$ & $(2,1)$ & $(2,2)$ \\
\hline$* A B A$ & $* A * B$ & $* B B A$ & $* B * B$ & $* * A A$ \\
\hline
\end{tabular}

There are two maximal sequences in the partial order: $T_{5}>T_{1}>T_{2}>T_{4}$ and $T_{5}>T_{1}>T_{3}>T_{4}$. The associated graded module $E_{0}^{p, q}$ is $E_{0}^{1, q}=\widetilde{U}_{5}, E_{0}^{2, q}=\widetilde{U}_{1}$, $E_{0}^{3, q}=\widetilde{U}_{2} \oplus \widetilde{U}_{3}, E_{0}^{4, q}=\widetilde{U}_{4}$, with $q$ determined by $p+q=i=u-2 v+2$. We show the $E_{1}, E_{2}$ and $E_{3}$ pages of the spectral sequence, which collapses at $E_{3}$. The generators and differentials are shown with $(u, v)$-gradings, which determine the $(p, q)$-gradings for the spectral sequence.
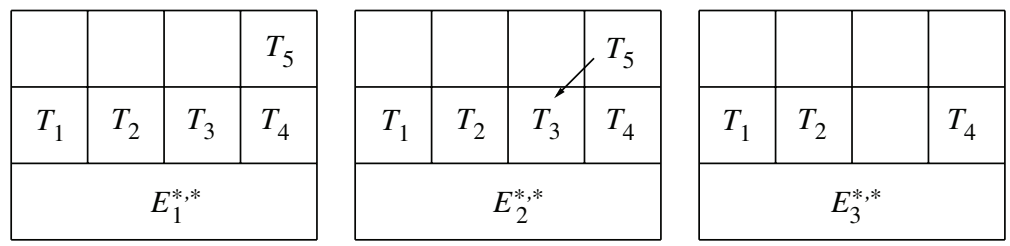

\section{ACKNOWLEDGMENTS}

We are grateful to Oleg Viro for many contributions. We thank the organizers of Knots in Poland 2003, and we also thank Alexander Shumakovitch and Peter Ozsváth for useful discussions.

\section{REFERENCES}

[1] M. Asaeda and J. Przytycki. Khovanov homology: torsion and thickness. In Advances in topological quantum field theory, volume 179 of NATO Sci. Ser. II Math. Phys. Chem., pages 135-166. Kluwer Acad. Publ., Dordrecht, 2004. MR2147419 (2006c:57008)

[2] A. Champanerkar, I. Kofman, and N. Stoltzfus. Graphs on surfaces and Khovanov homology. Algebr. Geom. Topol., 7:1531-1540, 2007. MR2366169

[3] M. Khovanov. A categorification of the Jones polynomial. Duke Math. J., 101(3):359-426, 2000. MR1740682(2002j:57025)

[4] M. Khovanov. Patterns in knot cohomology. I. Experiment. Math., 12(3):365-374, 2003. MR.2034399 (2004m:57022)

[5] E. S. Lee. An endomorphism of the Khovanov invariant. Adv. Math., 197(2):554-586, 2005. MR2173845 (2006g:57024)

[6] V. Manturov. Minimal diagrams of classical and virtual links, arXiv:math.GT/0501393.

[7] J. Rasmussen. Floer homology and knot complements, arXiv:math.GT/0306378.

[8] A. Shumakovitch. Torsion of the Khovanov homology, arXiv:math.GT/0405474.

[9] M. Thistlethwaite. A spanning tree expansion of the Jones polynomial. Topology, 26(3):297309, 1987. MR899051 (88h:57007)

[10] P. Traczyk. A combinatorial formula for the signature of alternating diagrams. Fund. Math., 184:311-316, 2004. MR2128055 (2005k:57020)

[11] O. Viro. Khovanov homology, its definitions and ramifications. Fund. Math., 184:317-342, 2004. MR2128056 (2006d:57024) 
[12] O. Viro. Remarks on definition of Khovanov homology, arXiv:math.GT/0202199.

[13] S. Wehrli. A spanning tree model for Khovanov homology, arXiv:math.GT/0409328.

Department of Mathematics, College of Staten Island, The City University of New York, Staten Island, New York 10314

E-mail address: abhijit@math.csi.cuny.edu

Department of Mathematics, College of Staten Island, The City University of New York, Staten Island, New York 10314

E-mail address: ikofman@math.csi.cuny.edu 